\title{
ON $m$ TH-COMMUTATORS AND ANTI-COMMUTATORS INVOLVING GENERALIZED DERIVATIONS IN PRIME RINGS
}

\author{
Mohd Arif Raza
}

(C) 2019 by University of Niš, Serbia | Creative Commons Licence: CC BY-NC-ND

\begin{abstract}
In this paper, we study the $m^{\text {th }}$-commutator and anti-commutator involving generalized derivations on some suitable subsets of rings. We attain the information about the structure of rings and the behaviour of the generalized derivation in the form of multiplication by some specific element of the Utumi quotient ring which satisfies certain differential identities.
\end{abstract}

Keywords: prime ring; Generalized derivation, Generalized polynomial identity.

\section{Motivation}

It was shown by Herstein [10] that if $d$ is a nonzero derivation of $\mathcal{R}$, a prime ring with a characteristic different from 2 such that $[d(x), d(y)]=0$ for all $x, y \in \mathcal{R}$, then $\mathcal{R}$ is commutative. Later, Bell and Daif [5] proved that if $\mathcal{R}$ is a semiprime ring, $\mathcal{I}$ is a nonzero right ideal of $\mathcal{R}$ and $d$ is a derivation of $\mathcal{R}$ such that $[d(x), d(y)]=[x, y]$ for all $x, y \in \mathcal{I}$, then $\mathcal{I} \subseteq \mathcal{Z}(\mathcal{R})$. Motivated by the above result, Huang [11] obtained the commutativity of prime ring $\mathcal{R}$ with characteristic different from 2 satisfies $[d(x), d(y)]_{m}=[x, y]^{n}$, for all $x, y \in \mathcal{I}$, a nonzero ideal of $\mathcal{R}$, where $1 \leq m, n \in$ $\mathbb{Z}^{+}$. In [2], Ashraf and Rehman studied anti-commutator involving derivation, i.e., $d(x) \circ d(y)=x \circ y$ and obtained the same conclusion.

On the other hand, Daif and Bell [7] proved that if $\mathcal{R}$ is a semiprime ring and $d$ is a nonzero derivation of $\mathcal{R}$ such that $d([x, y])=[x, y]$ for all $x, y \in \mathcal{R}$, then $\mathcal{R}$ is commutative. In this direction, Ashraf and Rehman [2] discussed the commutativity of prime ring $\mathcal{R}$ whenever $\mathcal{R}$ satisfies $d(x \circ y)=x \circ y$ for all $x, y \in \mathcal{I}$, a nonzero ideal of $\mathcal{R}$. In recent years, several algebraist studied various generalizations of above mentioned identities and obtained the structure of rings and behaviour of derivations and generalized derivations on rings (see $[1,8,12,19,20,21]$ and references therein).

Received November 12, 2018; accepted March 12, 2019

2010 Mathematics Subject Classification. Primary 16N20; Secondary 16W25 
In this note we shall examine the action of derivations and generalized derivations having $m$-th commutator and anti-commutator on prime rings. More precisely, we study the differential identities which involves both commutator and anticommutator on some appropriate subset of rings and obtain the information about the structure of rings and the behaviour of generalized derivation in the form of multiplication by some specific element of Utumi quotient ring.

Throughout this note, unless specifically stated, $\mathcal{R}$ denotes a prime ring, i.e., for $a, b \in \mathcal{R}, a \mathcal{R} b=(0)$ implies that either $a=0$ or $b=0$. A ring $\mathcal{R}$ is said to be a left (right) faithful ring if for $a \in \mathcal{R}, a \mathcal{R}=(0)(\mathcal{R} a=(0)$ resp.) implies $a=0$. For a left faithful ring $\mathcal{R}$, the right Utumi quotient ring of $\mathcal{R}$ can be characterized as the ring $\mathcal{U}_{r}(\mathcal{R})$ (up to isomorphisms fixing $\mathcal{R}$ ) satisfying the following properties: (1) $\mathcal{R}$ is a subring of $\mathcal{U}_{r}(\mathcal{R}) ;(2)$ For each $a \in \mathcal{U}_{r}(\mathcal{R})$, there exists a dense right ideal $\rho$ of $\mathcal{R}$ such that $a \rho \subseteq \mathcal{R}$; (3) If $a \in \mathcal{U}_{r}(\mathcal{R})$ and $a \rho=0$ for some dense right ideal $\rho$ of $\mathcal{R}$, then $a=0$; (4) For any dense right ideal $\rho$ of $\mathcal{R}$ and for any right $\mathcal{R}$-module map $\varphi: \rho_{\mathcal{R}} \rightarrow \mathcal{R}_{\mathcal{R}}$, there exists $a \in \mathcal{U}_{r}(\mathcal{R})$ such that $\varphi(x)=a x$ for all $x \in \rho$. Analogously, for a right faithful ring $\mathcal{R}$ we may define $\mathcal{U}_{l}(\mathcal{R})$ the left Utumi quotient ring of $\mathcal{R}$ in terms of dense left ideals of $\mathcal{R}$. Let $\mathcal{R}$ be a left and right faithful ring. The two-sided Utumi quotient $\operatorname{ring} \mathcal{U}$ of $\mathcal{R}$ is the subring of $\mathcal{U}_{r}(\mathcal{R})$ defined as follows: $\mathcal{U}=\left\{x \in U_{r}(\mathcal{R}) \mid \lambda x \subseteq \mathcal{R}\right.$ for some dense left ideal $\lambda$ of $\left.\mathcal{R}\right\}$. In [6, Theorem 2], Chuang proved that if $\mathcal{R}$ is a prime ring, then each dense right ideal and $\mathcal{U}$ satisfy the same generalized polynomial identities (GPIs) with coefficients in $\mathcal{U}$. In any case, when $\mathcal{R}$ is a prime ring, all we need about $\mathcal{U}$ is that $(1) \mathcal{R} \subseteq \mathcal{U}$; (2) $\mathcal{U}$ is a prime ring; (3) The center of $\mathcal{U}$, denoted by $\mathcal{C}$, is a field which is called the extended centroid of $\mathcal{R}$. The axiomatic formulations and the properties of this quotient ring $\mathcal{U}$ can be found in [3]. For any $x, y \in \mathcal{R}$, the symbol $[x, y]$ and $x \circ y$ stands for the commutator $x y-y x$ and anti-commutator $x y+y x$, respectively. we set $x \circ_{0} y=x, x \circ_{1} y=x \circ y=x y+y x$, and inductively $x \circ_{m} y=\left(x \circ_{m-1} y\right) \circ y$ for $m>1$. Again we set $[x, y]_{0}=x,[x, y]_{1}=[x, y]=x y-y x$ and inductively $[x, y]_{m}=\left[[x, y]_{m-1}, y\right]$ for $m>1$. An additive mapping $d: \mathcal{R} \rightarrow \mathcal{R}$ is called a derivation on $\mathcal{R}$ if $d(x y)=d(x) y+x d(y)$ holds for all $x, y \in R$. In particular, $d$ is an inner derivation induced by an element $q \in \mathcal{R}$ if $d(x)=[q, x]$ holds for all $x \in \mathcal{R}$. An additive mapping $F: \mathcal{R} \rightarrow \mathcal{R}$ is called generalized derivation of $\mathcal{R}$ if there exists a derivation $d$ of $\mathcal{R}$ such that $F(x y)=F(x) y+x d(y)$ for all $x, y \in R$.

\section{Main results}

We begin our discussion with the following remark as it is very crucial in developing the proof for our main results.

Remark 2.1. ([4, Lemma 7.1 ]) Let ${ }_{\mathcal{D}} \mathcal{M}$ be a left vector space over a division ring $\mathcal{D}$ with $\operatorname{dim}_{\mathcal{D}} \mathcal{M} \geq 2$ and $\mathcal{T} \in \operatorname{End}(\mathcal{M})$. If $x$ and $\mathcal{T} x$ are $\mathcal{D}$-dependent for every $x \in \mathcal{M}$, then there exists $\lambda \in \mathcal{D}$ such that $\mathcal{T} x=\lambda x$ for all $x \in \mathcal{M}$.

Now we prove our main results. 
Theorem 2.1. Let $1 \leq m, n \in \mathbb{Z}^{+}$. Next, let $\mathcal{R}$ be a prime ring of characteristic different from $2, \mathcal{I}$ be a nonzero ideal of $\mathcal{R}$ and $F$ be a nonzero generalized derivation associated with a derivation $d$ of $R$. If $F\left([x, y]_{m}\right)=d(x) \circ_{n} d(y)$ for all $x, y \in \mathcal{I}$, then either $R$ is commutative or $d=0$ and there exist $a \in \mathcal{U}$ such that $F(x)=a x$ for all $x \in R$.

Proof. By [16, Theorem 3], there exists an element $a \in \mathcal{U}$ and a derivation $d$ on $\mathcal{U}$ such that $F(x)=a x+d(x)$ for all $x \in \mathcal{R}$. In view of our hypothesis, we have $a\left([x, y]_{m}\right)+d\left([x, y]_{m}\right)=d(x) \circ_{n} d(y)$ which is rewritten as

$$
\begin{aligned}
a\left([x, y]_{m}\right) & +\sum_{k=1}^{m}(-1)^{k}\left(\begin{array}{c}
m \\
k
\end{array}\right)\left(\sum_{i+j=k-1} y^{i} d(y) y^{j}\right) x y^{m-k} \\
& +\sum_{k=0}^{m}(-1)^{k}\left(\begin{array}{c}
m \\
k
\end{array}\right) y^{k} d(x) y^{m-k} \\
& +\sum_{k=0}^{m-1}(-1)^{k}\left(\begin{array}{c}
m \\
k
\end{array}\right) y^{k} x\left(\sum_{r+s=m-k-1} y^{r} d(y) y^{s}\right)=d(x) \circ_{n} d(y)
\end{aligned}
$$

for all $x, y \in \mathcal{I}$. In the light of Kharchenko's theory [14], we split our proof into two cases. Firstly, we assume that $d$ is an $\mathcal{U}$-inner derivation induced by an element $q \in \mathcal{U}$, i.e., $d(x)=[q, x]$ for all $x \in \mathcal{R}$, then we have $a\left(x \circ_{m} y\right)+\left[q, x \circ_{m} y\right]=$ $[[q, x],[q, y]]_{n}$ for all $x, y \in \mathcal{I}$. By Chuang $[6$, Theorem 1], the last identity is also satisfied by $\mathcal{U}$. If $q \in C$, then $a\left(x \circ_{m} y\right)+\left[q, x \circ_{m} y\right]=[[q, x],[q, y]]_{n}$ reduces to $a\left(x \circ_{m} y\right)=0$ for all $x, y \in \mathcal{U}$. This a polynomial identity and by Lanski[15, Lemma 2], there exists a field $\mathbb{F}$ such that $\mathcal{U} \subseteq \mathcal{M}_{k}(\mathbb{F})$, the ring of $k \times k$ matrices over a field $\mathbb{F}$, where $k \geq 1$. Moreover, $\mathcal{U}$ and $\mathcal{M}_{k}(\mathbb{F})$ satisfy the same polynomial identity[15, Lemma 1], i.e., $a\left(x \circ_{m} y\right)=0$ for all $x, y \in \mathcal{M}_{k}(\mathbb{F})$. Now, we assuming $x=e_{12}$ and $y=e_{22}$, we have $0=a e_{12}$ which implies that $a_{11}=a_{21}=0$. Similarly, assuming $x=e_{21}$ and $y=e_{11}$ we can prove that $a_{22}=a_{12}=0$, i.e., $a=0$. Thus in all, $a\left(x \circ_{m} y\right)+\left[q, x \circ_{m} y\right]=[[q, x],[q, y]]_{n}$ is a non-trivial generalized polynomial identity (GPI) as $q \notin \mathcal{C}$. If the center $\mathcal{C}$ of $\mathcal{U}$ is infinite, then we have $a\left(x \circ_{m} y\right)+\left[q, x \circ_{m} y\right]=[[q, x],[q, y]]_{n}$ for all $x, y \in \mathcal{U} \otimes_{\mathcal{C}} \overline{\mathcal{C}}$, where $\overline{\mathcal{C}}$ is algebraic closure of $\mathcal{C}$. Since both $\mathcal{U}$ and $\mathcal{U} \otimes_{\mathcal{C}} \overline{\mathcal{C}}$ are prime and centrally closed $[9$, Theorem 2.5 and Theorem 3.5], we may replace $\mathcal{R}$ by $\mathcal{U}$ or $\mathcal{U} \otimes_{\mathcal{C}} \overline{\mathcal{C}}$ according as $\mathcal{C}$ is finite or infinite. Thus, we may assume that $\mathcal{R}$ is centrally closed over $\mathcal{C}$ (i.e., $\mathcal{R C}=\mathcal{R}$ ) which is either finite or algebraically closed and $a\left(x \circ_{m} y\right)+\left[q, x \circ_{m} y\right]=[[q, x],[q, y]]_{n}$ for all $x, y \in \mathcal{R}$. By Martindale [17, Theorem 3], $\mathcal{R C}$ (and so $\mathcal{R}$ ) is a primitive ring having nonzero socle $\mathcal{H}$ with $\mathcal{C}$ as the associated division ring. Hence, by Jacobson's theorem [13, p.75], $\mathcal{R}$ is isomorphic to a dense ring of linear transformations of some vector space $\mathcal{V}$ over $\mathcal{C}$ and $\mathcal{H}$ consists of finite rank linear transformations in $\mathcal{R}$. If $\mathcal{V}$ is finite dimensional over $\mathcal{C}$, then the density of $\mathcal{R}$ on $\mathcal{V}$ implies that $\mathcal{R} \cong \mathcal{M}_{m}(\mathcal{C})$, where $m=\operatorname{dim}_{\mathcal{C}} \mathcal{V}$.

Suppose that $\operatorname{dim}_{\mathcal{C}} \mathcal{V} \geq 3$ such that $v$ and $q v$ are linearly $\mathcal{C}$-independent for all $v \in \mathcal{V}$. By density of $\mathcal{R}$, there exists $u \in \mathcal{V}$ such that $v, q v$ and $u$ are linearly 
$\mathcal{C}$-independent and $x, y \in \mathcal{R}$ such that

$$
\begin{aligned}
& x v=0, \quad x q v=-u, \quad x u=v, \quad x q u=0 \\
& y v=0, \quad y q v=-v, \quad y u=0, \quad y q u=-u .
\end{aligned}
$$

Applying density theorem, we see that

$$
0=\left(a\left([x, y]_{m}\right)+[q, x] \circ_{m}[q, y]-\left[q,[x, y]_{n}\right]\right) v=2^{m} u
$$

a contradiction, as $\operatorname{char}(\mathcal{R}) \neq 2$. Hence, we conclude that $\{v, q v\}$ is linearly $\mathcal{C}$ dependent for all $v \in \mathcal{V}$. Thus, by Remark 2.1, there exists $\lambda \in \mathcal{C}$ such that $q v=v \lambda$ for any $v \in \mathcal{V}$.

For $r \in \mathcal{R}, v \in \mathcal{V}$, we can write, $q v=v \lambda, r(q v)=r(v \lambda)$, and also $q(r v)=(r v) \lambda$. Thus $0=[q, r] v$ for any $v \in \mathcal{V}$, i.e., $[q, r] \mathcal{V}=0$. Since $\mathcal{V}$ is a left faithful irreducible $\mathcal{R}$-module, we have $[q, r]=0$ for all $r \in \mathcal{R}$, i.e., $q \in Z(\mathcal{R})$ which gives $d=0$ and hence $F(x)=a x$ for all $x \in \mathcal{R}$.

Now suppose that $\operatorname{dim}_{\mathcal{C}} \mathcal{V} \leq 2$. In this case $\mathcal{R}$ is a simple GPI-ring with 1 and so it is a central simple algebra finite dimensional over its center. By Lanski[15, Lemma 2], it follows that there exists a suitable field $\mathbb{F}$ such that $\mathcal{R} \subseteq \mathcal{M}_{m}(\mathbb{F})$ the ring of $m \times m$ matrices over $\mathbb{F}$ and moreover, $\mathcal{M}_{m}(\mathbb{F})$ satisfy the same GPI as $\mathcal{R}$. Assume $m \geq 3$, then by the same argument as above we get the conclusion. Obviously if $m=1$, then $\mathcal{R}$ is commutative. Thus we may assume that $m=2$, i.e., $\mathcal{R} \subseteq \mathcal{M}_{2}(\mathbb{F})$, where $\mathcal{M}_{2}(\mathbb{F})$ satisfies $a\left([x, y]_{m}\right)+[q, x] \circ_{m}[q, y]-\left[q,[x, y]_{n}\right]=$ 0 . Denote by $e_{i j}$ the usual unit matrix with 1 at $(i, j)$-entry and zero elsewhere. By putting $x=y=e_{12}$ and $q=\sum_{i, j} q_{i j} e_{i j}$ in the above identity and then right multiplying by $e_{12}$, one can easily get $\left(e_{12} q\right)^{m+1} e_{12}=0$. It follows easily that $\left(\begin{array}{cc}0 & q_{21}^{m+1} \\ 0 & 0\end{array}\right)=0$ implies that $q_{21}=0$. Similarly we can get $q_{12}=0$. Thus in all, we see that $q$ is a diagonal matrix in $\mathcal{M}_{2}(\mathbb{F})$. Let $\psi \in \operatorname{Aut}\left(\mathcal{M}_{2}(\mathbb{F})\right)$. Since $\psi(a)\left([\psi(x), \psi(y)]_{m}\right)+[\psi(q), \psi(x)] \circ_{m}[\psi(q), \psi(y)]-\left[\psi(q),[\psi(x), \psi(y)]_{n}\right]=0, \psi(q)$ must be a diagonal matrix in $\mathcal{M}_{2}(\mathbb{F})$. In particular, let $\psi(x)=\left(1-e_{i j}\right) x\left(1+e_{i j}\right)$ for $i \neq j$. Then $\psi(q)=q+\left(q_{i i}-q_{j j}\right) e_{i j}$, i.e., $q_{i i}=q_{j j}$ for $i \neq j$. This implies that $q$ is central in $\mathcal{M}_{2}(\mathbb{F})$, which leads to $d=0$. Now lastly, we assume that $d$ is $\mathcal{U}$-outer derivation, then $\mathcal{I}$ satisfies the polynomial identity

$$
\begin{aligned}
a\left([x, y]_{m}\right) & +\sum_{k=1}^{m}(-1)^{k}\left(\begin{array}{c}
m \\
k
\end{array}\right)\left(\sum_{i+j=k-1} y^{i} z y^{j}\right) x y^{m-k}+\sum_{k=0}^{m}(-1)^{k}\left(\begin{array}{c}
m \\
k
\end{array}\right) y^{k} w y^{m-k} \\
& +\sum_{k=0}^{m-1}(-1)^{k}\left(\begin{array}{c}
m \\
k
\end{array}\right) y^{k} x\left(\sum_{r+s=m-k-1} y^{r} z y^{s}\right)=w \circ_{n} z
\end{aligned}
$$

for all $x, y, z, w \in \mathcal{I}$. In particular, if we take $x=z=0$, then $\mathcal{I}$ satisfies the polynomial identity

$$
\sum_{k=0}^{m}(-1)^{k}\left(\begin{array}{c}
m \\
k
\end{array}\right) y^{k} w y^{m-k}=0
$$


for all $y, w \in \mathcal{I}$. That is, $[w, y]_{m}=0$ for all $w, y \in \mathcal{I}$, which can be written as $\left[\mathcal{I}_{w}(y), y\right]_{m-1}=0$ for all $w, y \in \mathcal{I}$, where $\mathcal{I}_{w}(y)$ is an inner derivation determined by w. By Lanski [15, Theorem 1], either $R$ is commutative or $\mathcal{I}_{w}=0$ i.e., $\mathcal{I} \subseteq Z(\mathcal{R})$ in which case $\mathcal{R}$ is also commutative by Mayne [18, Lemma 3].

Theorem 2.2. Let $1 \leq m, n \in \mathbb{Z}^{+}$. Next, let $\mathcal{R}$ be a prime ring of characteristic different from $2, \mathcal{I}$ be a nonzero ideal of $\mathcal{R}$ and $F$ be a nonzero generalized derivation associated with a derivation $d$ of $R$. If $F\left(x \circ_{m} y\right)=[d(x), d(y)]_{n}$ for all $x, y \in \mathcal{I}$, then either $R$ is commutative or $d=0$ and there exists $a \in \mathcal{U}$ such that $F(x)=a x$ for all $x \in \mathcal{R}$.

Proof. By the given hypothesis and [16, Theorem 3], we have $a\left(x \circ_{m} y\right)+d\left(x \circ_{m} y\right)=$ $[d(x), d(y)]_{n}$ which is rewritten as

$$
\begin{aligned}
a\left(x \circ_{m} y\right) & +\sum_{k=1}^{m}\left(\begin{array}{c}
m \\
k
\end{array}\right)\left(\sum_{i+j=k-1} y^{i} d(y) y^{j}\right) x y^{m-k}+\sum_{k=0}^{m}\left(\begin{array}{c}
m \\
k
\end{array}\right) y^{k} d(x) y^{m-k} \\
& +\sum_{k=0}^{m-1}\left(\begin{array}{c}
m \\
k
\end{array}\right) y^{k} x\left(\sum_{r+s=m-k-1} y^{r} d(y) y^{s}\right)=[d(x), d(y)]_{n}
\end{aligned}
$$

for all $x, y \in \mathcal{I}$. In view of Kharchenko's theory [14], we divide the proof into two cases:

Case 1. If $d$ is $\mathcal{U}$-outer, then $\mathcal{I}$ satisfies the polynomial identity

$$
\begin{aligned}
a(x \circ m y) & +\sum_{k=1}^{m}\left(\begin{array}{c}
m \\
k
\end{array}\right)\left(\sum_{i+j=k-1} y^{i} z y^{j}\right) x y^{m-k}+\sum_{k=0}^{m}\left(\begin{array}{c}
m \\
k
\end{array}\right) y^{k} w y^{m-k} \\
& +\sum_{k=0}^{m-1}\left(\begin{array}{c}
m \\
k
\end{array}\right) y^{k} x\left(\sum_{r+s=m-k-1} y^{r} z y^{s}\right)=[w, z]_{n}
\end{aligned}
$$

for all $x, y, z, w \in \mathcal{I}$. In particular if we take $x=z=0$, then $\mathcal{I}$ satisfies the polynomial identity

$$
\sum_{k=0}^{m}\left(\begin{array}{c}
m \\
k
\end{array}\right) y^{k} w y^{m-k}=0
$$

for all $y, w \in \mathcal{I}$. That is $w \circ_{m} y=0$ for all $w, y \in \mathcal{I}$. Using the same argument as used in Theorem 2.1 and by choosing $w=e_{12}, y=e_{11}$, we see that $w \circ_{m} y=e_{12} \neq 0$, a contradiction.

Case 2. If $d$ is $\mathcal{U}$-inner derivation induced by an element $q \in \mathcal{U}$, i.e., $d(x)=[q, x]$ for all $x \in \mathcal{R}$, then we have $a\left(x \circ_{m} y\right)+\left[q, x \circ_{m} y\right]=[[q, x],[q, y]]_{n}$ for all $x, y \in \mathcal{I}$. By Chuang [6, Theorem 1], $\mathcal{I}$ and $\mathcal{U}$ satisfy same generalized polynomial identities (GPIs), i.e., $a\left(x \circ_{m} y\right)+\left[q, x \circ_{m} y\right]=[[q, x],[q, y]]_{n}$ for all $x, y \in \mathcal{U}$. Using the similar techniques with necessary variations as used in the proof of Theorem 2.1, 
we see that if $\mathcal{V}$ is finite dimensional over $\mathcal{C}$, then the density of $\mathcal{R}$ on $\mathcal{V}$ implies that $\mathcal{R} \cong \mathcal{M}_{m}(\mathcal{C})$, where $m=\operatorname{dim}_{\mathcal{C}} \mathcal{V}$.

Suppose that $\operatorname{dim}_{\mathcal{C}} \mathcal{V} \geq 2$. Now, we want to show that $v$ and $q v$ are linearly $\mathcal{C}$-dependent for all $v \in \mathcal{V}$. If $q v=0$, then $\{v, q v\}$ is linearly $\mathcal{C}$-dependent. Suppose on the contrary that $v$ and $q v$ are linearly $\mathcal{C}$-independent for some $v \in \mathcal{V}$.

If $q^{2} v \notin \operatorname{Span}_{\mathcal{C}}\{v, q v\}$, then the set $\left\{v, q v, q^{2} v\right\}$ is linearly $\mathcal{C}$-independent. Since $v$ and $q v$ are linearly $\mathcal{C}$-independent, by the density of $\mathcal{R}$, there exist $x, y \in \mathcal{R}$ such that

$$
\begin{aligned}
& x v=v, \quad x q v=0, \quad x q^{2} v=0 ; \\
& y v=0, \quad y q v=-v, \quad y q^{2} v=0 .
\end{aligned}
$$

When $m=n=1$, then we see that

$$
0=\left(a\left(x \circ_{m} y\right)+\left[q, x \circ_{m} y\right]-[[q, x],[q, y]]_{n}\right) v=2 q v-v .
$$

Moreover, when $m, n>1$, we have

$$
0=\left(a\left(x \circ_{m} y\right)+\left[q, x \circ_{m} y\right]=[[q, x],[q, y]]_{n}\right) v=2^{m} q v
$$

In both the cases we get a contradiction as characteristic of $\mathcal{R}$ is different from 2 .

If $q^{2} v \in \operatorname{Span}_{\mathcal{C}}\{v, q v\}$, then $q^{2} v=v \beta+q v \gamma$ for some $\beta, \gamma \in \mathcal{C}$. By the density of $\mathcal{R}$, there exist $x, y \in \mathcal{R}$ such that

$$
\begin{aligned}
& x v=v, \quad x q v=0 \\
& y v=0, \quad y q v=-v .
\end{aligned}
$$

For this, first we take $m=n=1$, we see that

$$
0=\left(a\left(x \circ_{m} y\right)+\left[q, x \circ_{m} y\right]-[[q, x],[q, y]]_{n}\right) v=2 q v-v \gamma-v .
$$

Now, when $m, n>1$, we have

$$
0=\left(a\left(x \circ_{m} y\right)+\left[q, x \circ_{m} y\right]-[[q, x],[q, y]]_{n}\right) v=2^{m} q v-2^{m-1} v \gamma
$$

Using an argument similar to that mentioned above, we get a contradiction in both cases. So, we conclude that $\{v, q v\}$ is linearly $\mathcal{C}$-dependent for all $v \in \mathcal{V}$. Thus, by Remark 2.1, there exists $\lambda \in \mathcal{C}$ such that $q v=v \lambda$ for any $v \in \mathcal{V}$.

For $r \in \mathcal{R}, v \in \mathcal{V}$, we can write, $q v=v \lambda, r(q v)=r(v \lambda)$, and also $q(r v)=(r v) \lambda$. Thus $0=[q, r] v$ for any $v \in \mathcal{V}$, i.e., $[q, r] \mathcal{V}=0$. Since $\mathcal{V}$ is a left faithful irreducible $\mathcal{R}$-module, we have $[q, r]=0$ for all $r \in \mathcal{R}$, i.e., $\quad q \in Z(\mathcal{R})$ and hence $d=0$. This completes the proof.

In view of Theorem 2.1 and Theorem 2.2, we can write the following corollaries (proofs are omitted for sake of brevity) 
Corollary 2.1. Let $1 \leq m \in \mathbb{Z}^{+}$. Next, let $\mathcal{R}$ be a prime ring of a characteristic different from $2, \mathcal{I}$ be a nonzero ideal of $\mathcal{R}$ and $d$ be a derivation of $R$. If $d(x) \circ_{m}$ $d(y)=0$ for all $x, y \in \mathcal{I}$, then either $R$ is commutative or $d=0$.

Corollary 2.2. Let $1 \leq m \in \mathbb{Z}$. Next, let $\mathcal{R}$ be a prime ring of a characteristic different from $2, \mathcal{I}$ be a nonzero ideal of $\mathcal{R}$ and $d$ be a derivation of $R$. If $[d(x) \circ$ $d(y)]_{m}=0$ for all $x, y \in \mathcal{I}$, then either $R$ is commutative or $d=0$.

Acknowledgements: The author would like to thank the referee for his/her valuable suggestions.

\section{R E F E R E N C E S}

1. N. ArgaÇ and H. G. Inceboz: Derivation of prime and semiprime rings. J. Korean Math. Soc., 46 (5) (2009), 997-1005.

2. M. Ashraf and N. Rehman: On commutativity of rings with derivations. Results Math., 42 (1-2) (2002), 3-8.

3. K. I. Beidar, W. S. Martindale III and A. V. Mikhalev: Rings with Generalized Identities. Pure and Applied Mathematics, Marcel Dekker 196, New York, 1996.

4. K. I. BEIDAR and M. BRE s̆AR: Extended Jacobson density theorem for rings with automorphisms and derivations, Israel J. Math., 122 (2001), 317-346.

5. H. E. BELL and M. N. DAIF: On commutativity and strong commutativity-preserving maps. Cand. Math. Bull., 37 (1994), 443-447.

6. C. L. Chuang: GPIs having coefficients in Utumi quotient rings. Proc. Amer. Math. Soc. 103 (1988), 723-728.

7. M. N. DAIF and H. E. BELL: Remarks on derivations on semiprime rings. Internt. J. Math. Math. Sci. 15 (1992), 205-206.

8. Q. DENG and M. AsHRAF:On strong commutativity preserving mappings. Results Math. 30 (1996), 259-263.

9. T. S. Erickson, W. S. Martindale III and J. M. Osborn: Prime nonassociative algebras. Pacific. J. Math. 60 (1975), 49-63.

10. I. N. Herstein: A note on derivations. Canad. Math. Bull. 21 (1978), 241-242.

11. S. HuAng: Derivation with Engel conditions in prime and semiprime rings. Czechoslovak Math. J. 61 (136)(2011), 1135-1140.

12. S. HuAng: Generalized derivations of prime and semiprime rings. Taiwanese J. Math., 16 (2) (2012), 771-776.

13. N. Jacobson: Structure of Rings. Colloquium Publications 37, Amer. Math. Soc. VII, Provindence, RI 1956.

14. V. K. Kharchenko: Differential identities of prime rings. Algebra Logic 17 (1979), 155-168.

15. C. Lanski: An Engel condition with derivation. Proc. Amer. Math. Soc. 118 (1993), 731-734. 
16. T. K. LEE: Generalized derivations of left faithful rings. Comm. Algebra 27 (8)(1998), 4057-4073.

17. W. S. MARTINDAle III: Prime rings satisfying a generalized polynomial identity. J. Algebra 12 (1969), 576-584.

18. J. H. MAYne: Centralizing mappings of prime rings. Canad. Math. Bull. 27 (1) (1984), $122-126$.

19. M. A. RAZA and N. REHMAN: On prime and semiprime rings with generalized Derivations and non-commutative Banach algebras. Proc. Indian Acad. Sci. (Math. Sci.) 126 (3) (2016), 389-398.

20. M. A. RAZA and N. REHMAN: On generalized derivation in rings and Banach algebras. Kragujevac J. Math. 41 (1) (2017), 105-120.

21. N. Rehman, M. A. RAZA and T. BAno: On commutativity of rings with generalized derivations. J. Egyptian Math. Soc. 24 (2) (2016), 151-155.

\author{
Mohd Arif Raza \\ Department of Mathematics \\ Faculty of Science \& Art-Rabigh \\ King Abdulaziz University \\ Jeddah KSA \\ arifraza03@gmail.com
}

